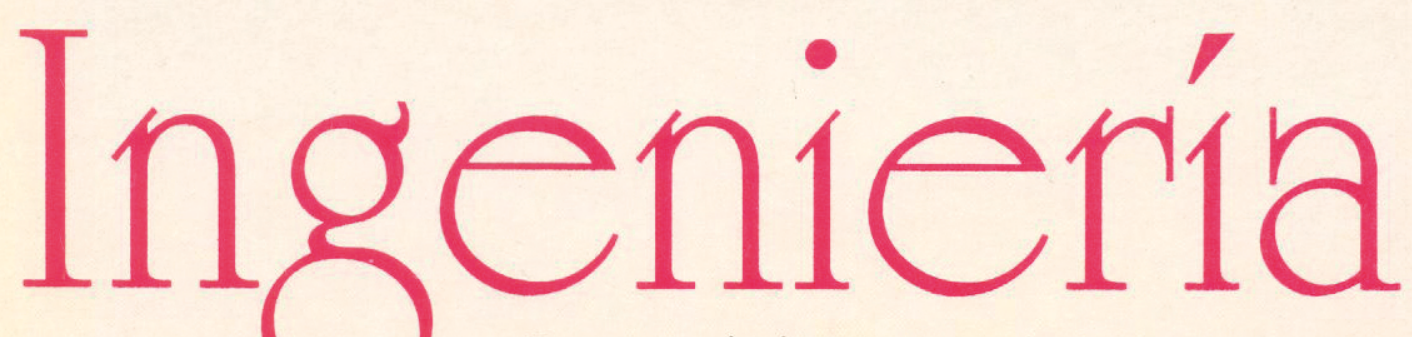

Revista de la Universidad de Costa Rica ENERO/JUNIO 1994 VOLUMEN 4 N 1

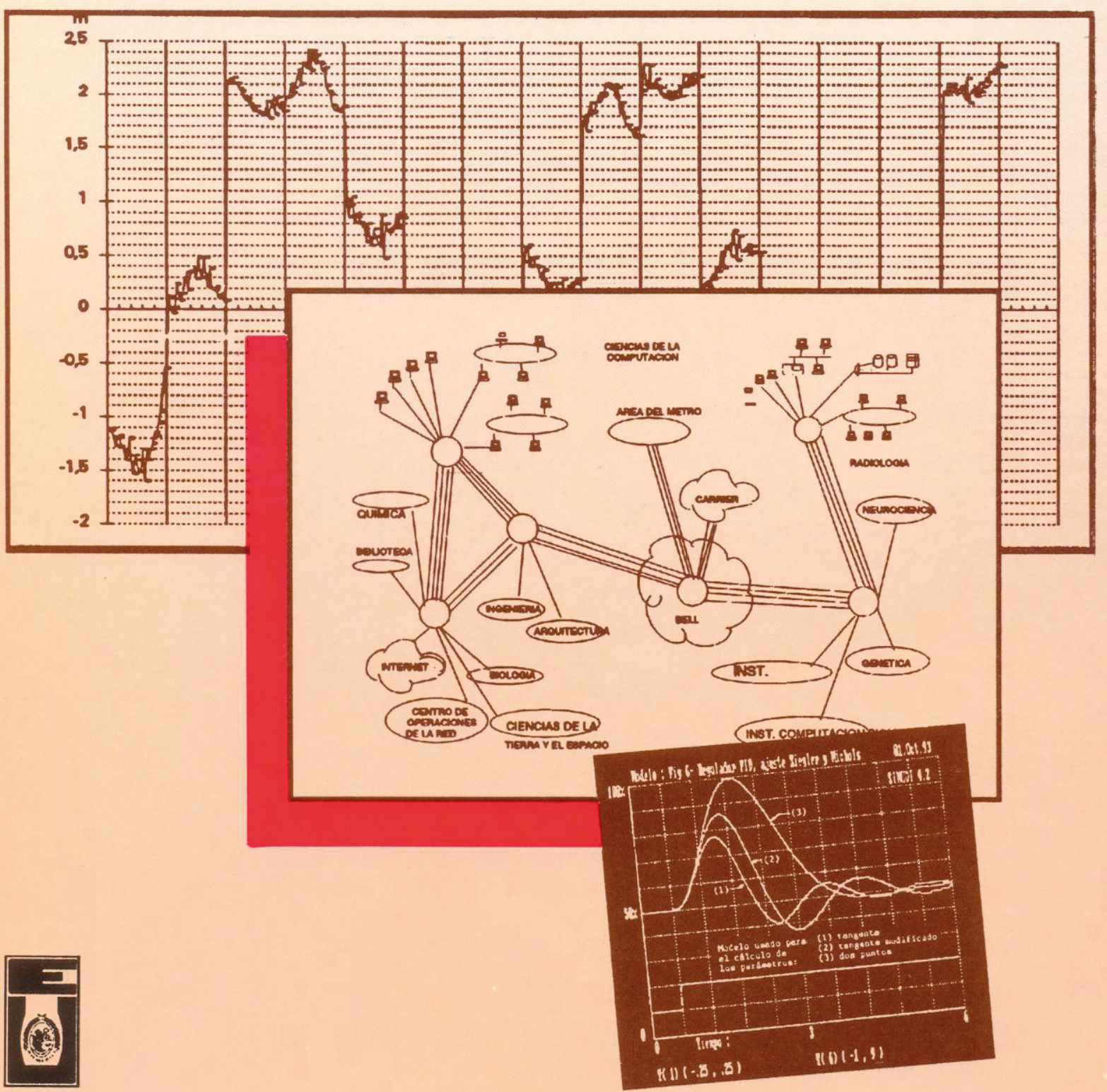




\title{
DESVANECIMIENTO POR MULTITRAYECTORIAS EN ENLACES DE MICROONDAS
}

\begin{abstract}
RESUMEN
Los desvanecimientos debido a trayectorias múhiples en enlaces largos de microondas analógicas y digitales en la mayoría de los casos son la condición que afecta mas significativamente el desempeño del enlace.

En este artículo se discutirá los fundamentos estadísticos del desvanecimiento plano tipo Rayleigh, que ha sido usado en el diseño de radioenlaces en nuestro ambiente.
\end{abstract}

\section{SUMMARY}

Muhipath fading in long digital and analong microwave link is in most cases, the major condition that affects overall system performance.

In this paper it will be discussed the probabilisticfundamentals oftheflat Rayleighfadingthat has been usedfor designing radio links in our enviromenl

\section{INTRODUCCIÓN}

El modelo de multitrayectorias de la señal recibida presupone que ésta se compone de la suma de varias señales que llegan al receptor por diferentes trayectos, exhibiendo una fase aleatoria y una magnitud Gaussiana. La composición vectorial de estas señales dan como resultado una señal de amplitud aleatoria con distribución Rayleigh.

Las causas que provocan esta dispersión de la señal, se deben a la estratificación de los índices de refracción de la atmósfera, los cuales son cambiantes durante todo el año.

Se sabe que este fenómeno no es estacionario. En algunas épocas del año la atmósfera se presenta altamente heterogénea; es decir, muy estratificada, en cuanto al indice de refracción se refiere y aunque el enlace sea "linea vista" y $\sin$ obstrucciones, aparecen en el receptor múltiples señales de las cuales no hay ninguna que presente una preponderancia sobre las otras.

Profesor de la Escuela de Ingeniería Eléctrica, Universidad de Costa Rica.
Durante el proceso de análisis se denotará como 11 la probabilidad de ocurrencia del desvanecimiento, siendo su duración más larga que la de un simbolo. Esta situación especial de la duración relativa del evento de desvanecimiento con respecto a la del simbolo se denomina desvanecimiento plano o lento.

\section{EL MODELO}

El receptor recibirá una señal que es el resultado de la composición de múltiples señales cuya amplitud es Gaussiana y la fase uniformemente distribuida en el intervalo de $\mathrm{O}$ a $27 \mathrm{t}$.

$R j(t)=L_{;=1}^{N} a ; \cos ($ ro $o t+<I>t+8 j)+n(t)$ donde:

$\mathrm{n}(\mathrm{t})$ es ruido blanco Gaussiano adicionado por el receptor.

$\sim 1$ es uniformemente distribuida en $(\mathrm{O}, 21 t)$

$8 \mathrm{j}$ es la fase del j-ésimo simbolo enviado en un sistema de modulación DQPSK.

$a ; \quad$ es la amplitud de la i-ésima señal recibida. 
La señal recibida puede representarse también de la siguiente forma:

$$
\begin{aligned}
& \operatorname{Rj}(1) \sim(\lfloor, a, \operatorname{COS} \$ 1) c 0\{\text { ro. } 1+9 j)- \\
& \left(\left\lfloor, \boldsymbol{d}_{\mathbf{g}} \operatorname{sen} \$ 1\right) \operatorname{sen}(\text { ro } .1+9 j)+n(l)\right.
\end{aligned}
$$

Para N suficientemente grande (ambiente dispersivo), cada una de las sumatorias puede considerarse como una variable aleatoria Gaussiana (Teorema del Límite Central), pudiéndose simplificar la expresión de la señal recibida asi:

$$
\begin{aligned}
R\}(t)= & \text { VICOs(ro ot }+8\})- \\
& \left.\mathrm{v}_{2} \operatorname{sen}(\text { ro } o t+8\}\right)+n(t)
\end{aligned}
$$

\section{FUNCIÓN DE DENSIDAD PROBABI- LÍSTICA DE LA SEÑAL RECffiIDA}

La presentación anterior se denomina comúnmente como representación en fase y cuadratura de la señal recibida.

Igualmente se puede representar en magnitud y fase de la siguiente forma:

$R j(t)=\frac{\| U_{i}+u_{2}}{\cos (\text { root }}+\underset{0}{\mathrm{Bj}+\sim)}+\mathrm{n}(\mathrm{t})$

Es fácil demostrar que si , 1 Y 2 son Gaussianas e independientes, entonces las nuevas variables aleatorias:

$$
\begin{aligned}
& \mathrm{R}=\stackrel{\sim \mathrm{UL}+\mathrm{O}_{2}^{-}}{ } \\
& \triangleleft=\operatorname{Tan}^{-1}\left(0_{1} 10 \mathrm{~J}\right.
\end{aligned}
$$

son, la primera Rayleigh y la segunda uniforme en el intervalo $[0,2], \quad \mathrm{Y}$ estadísticamente independientes, esto es:

$$
\mathrm{f}(\mathrm{R}, \sim)=\left(\mathrm{R} / 27 \mathrm{tcr} \mathrm{r}^{2}\right) \exp \left\{-\mathrm{R}^{2} 12 c r^{2}\right\}
$$

Se puede observar inmediatamente que:

$$
\begin{gathered}
\mathrm{f}\left(\mathrm{R},\langle\mathrm{I} \gg)=\left(\begin{array}{lll}
R l & \mathrm{cr} &
\end{array}\right) \exp \left\{\begin{array}{ll}
\mathrm{R}^{2} & \left.12 c r^{2}\right\}
\end{array}\right. \text { * }\right. \\
(1 / 21 \mathrm{t})=\mathrm{f}(\mathrm{R}) \quad \mathrm{f}(\langle\mathrm{l})\rangle
\end{gathered}
$$

Así que son independientes, y la función de densidad probabilística de la amplitud de la señal distribuida es Rayleigh y está dado por:

$$
\begin{gathered}
\mathrm{f}(\mathrm{R})=\left(\mathrm{R} I \mathrm{cr}^{2}\right) \quad \exp \left\{-\mathrm{R} 1 \quad 12 c r^{\prime}\right\} \\
0<\mathrm{R}<\mathrm{ex}>
\end{gathered}
$$

\section{CÁLCULO DE LA PROBABILIDAD DE DES VANECIMIENTO}

El desvanecimiento de la sefíal se da cuando, la sefíal recibida disminuye su nivel de recepción nominal de "espacio libre" a valores menores que este, durante un período de tiempo.

Así, la probabilidad de que la señal recibida sea menor que $X$, por debajo del nivel de señal en ausencia de desvanecimiento es:

Para valores grandes de $\mathrm{X}$, el valor asintótico de la expresión anterior es:

$$
\mathrm{P}[\mathrm{R}<\mathrm{X}] \cong\left(1 / 2 \sigma^{2}\right) \mathrm{X}
$$

Expresando este valor en dectbeles con respecto a la condición de no desvanecimiento:

$$
\mathrm{F}=-10 \log _{10} \mathrm{x}^{2}
$$

Obtenemos la expresión conocida:

$$
\mathrm{P}[\mathrm{R}<\mathrm{X}]=\left(1 / 2 \sigma^{2}\right) 10^{-\mathrm{F} / 10}
$$

donde $\mathrm{F}$ es desvanecimiento en $\mathrm{dB}$ que sufriría la señal, por debajo del valor de referencia de espacio libre.

Sea $\boldsymbol{\mu}$ el nivel para el cual la probabilidad de que la señal desvanecida este por encima o por debajo de ese valor sea $1 / 2$. 
Esto implica que:

$\mathrm{P}[\mathrm{R}<\mu]=1 \mathrm{I} 2=1-\exp \left\{\mu 2 \quad / 2 \mathrm{cr}^{2}\right\}$

de donde podemos concluir que el término $\left(1120^{\prime} 2\right)$ se puede representar como:

$$
\left(1 / 2\left(^{2}\right)=f n 2 / \mu^{2}\right.
$$

En decibeles:

$$
\mathrm{L}^{2}=- \text { IOloglo } \quad \mu 2
$$

Así:

$$
\mathrm{p}[\mathrm{R}<\mu]=f n 2 \cdot 10-(F-L) f I O
$$

Finalmente, si 11es la probabilidad de que el desvanecimiento ocurra, entonces la probabilidad de tener desvanecimientos por múltiples trayectorias sería:

$$
\operatorname{Pr}(\mathrm{F})=11 \mathrm{P}[\mathrm{R}<\mathrm{X}]=11 \text { fn2.1 O- }(\mathrm{F}-\mathrm{L}) / \mathrm{lO}
$$

$$
\operatorname{Pr}(F)=0.693 \quad 11|O-(F-L) f| O
$$

La expresión para el desvanecimiento por trayectorias múltiples que aparece comúnmente en la literatura es:

$$
\mathrm{Pr}=\mathrm{Po} \text { 10- } \mathrm{FflO}
$$

Por comparación se obtiene que:

$$
\mathrm{Po}=0.6931110 \mathrm{~L} / \mathrm{IO}
$$

Empíricamente se le ha dado a Po una relación funcional con el tipo de terreno, el clima, la frecuencia y la distancia del enlace:

\section{$\mathrm{Po}=0.3 \mathrm{ab}(\mathrm{f} / 4)(\mathrm{d} / 50 \mathrm{r}$}

a: coeficiente del clima, oscila entre $1 / 4$ y 4 .

b: coeficiente del terreno, oscila entre $1 / 4$ y 4 .

f: frecuencia en $\mathrm{GHz}$.

d: longitud del enlace en $\mathrm{Km}$.

\section{CONCLUSIÓN}

Se ha hecho una deducción de la expresión de la probabilidad de desvanecimiento debido a multitrayectorias desde sus fundamentos.

Al final del artículo se presenta una relación teórica en comparación con una empírica en la cual se relaciona la probabilidad de que ocurra un desvanecimiento, con condiciones geográficas y climatológicas del trayecto sobre el cual se esta realizando el enlace.

\section{BffiLIOGRAFIA}

BreipoW, Arthur; Probabilistic System Analysis ( John Wiley \& Sons, Inc., 1970)

Van Trees, Harry; Detection. Estimation and Modulation Theory, Part I (John Wiley \& Sons, Inc., 1968)

Lee, William C.; Mobile Communications Engineering_(Mc Graw Hill, Inc., 1982)

Giger, Adolf J.; Low-Angle Microwave Propagation (Artech House, 1991) 